\title{
PROCESSO DE MOAGEM DE GARRAFAS PET PÓS-CONSUMO
}

\section{MOLDING PROCESS POST-CONSUMPTION PET BOTTLE}

\author{
E. A. SILVA ${ }^{1 * *}$, V. F. C. LINS $^{1}$ and F. COTTING ${ }^{1}$
}

Universidade Federal de Minas Gerais, Departamento de Engenharia Química, Belo Horizonte, Minas Gerais, Brasil

Autor correspondente. Universidade Federal de Minas Gerais, Departamento de Engenharia Química, Belo Horizonte, Minas Gerais, Brasil, Phone: +55 31 34091737 endereço de email: silvaelisangelax@gmail.com (E.A. SILVA).

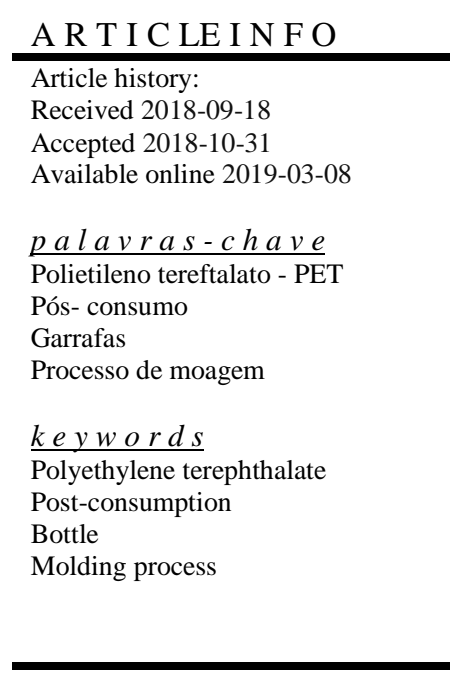

\section{RESUMO}

Atualmente, grandes volumes de lixo são descartados, contaminando e degradando o meio ambiente. Uma forma de remediação bastante difundida são as implementações de associações que incentivem a coleta seletiva de resíduos. A Associação Brasileira da Indústria de PET (ABIPET) acompanha a reciclagem e a reutilização de embalagens de PET no Brasil. Segundo estudos realizados, o volume de material descartado incorretamente ainda é superior ao volume produzido. O PET é um polímero de engenharia com excelentes propriedades $e$ com vasta aplicação. Após o processamento, grande parte das propriedades do PET são mantidas, neste sentido, a reutilização desse polímero como matéria prima é favorável. Porém, o PET reciclado é fornecido em forma de flakes o que limita a sua aplicação. A proposta deste trabalho é fornecer uma metodologia para moagem das garrafas PET pósconsumo com granulometria abaixo de $0,3 \mathrm{~mm}$ para ampliar a aplicabilidade do PET. $O$ material produzido foi caracterizado pelas técnicas de FTIR, DSC, TG e distribuição granulométrica.

A B S T R A C T

Currently, large bulk of waste are discarded therewith the environment is increasingly contaminated and degraded. One possibility is to increase the waste collection. The Brazilian Association of PET Industry (ABIPET) verified that incorrect disposal of PET packaging is still higher than bulk produced. PET is an engineering polymer with excellent properties and wide application. Most of properties are maintained after processing. Therefore, the reutilization of the polymer as secondary raw material is favorable. However, recycled PET is supplied in the form of flakes, which limits its application. This paper provides a methodology for milling the post-consumer PET bottles, obtaining a granulometry below $0.3 \mathrm{~mm}$ in order to increase its applicability. The material produced was characterized by the FTIR, DSC, TG and granulometric distribution techniques. 


\section{INTRODUCC̃̃O}

Segundo levantamento realizado pela Associação Brasileira de Empresas de Limpeza Pública e Resíduos Especiais (Abrelpe) em 2016 verificou-se que a população brasileira gera cerca de 78,3 milhões de toneladas de resíduos por ano (ABRELPE, 2016). Geralmente, o lixo é constituído de resíduos de construção e demolição, resíduo de serviço de saúde, resíduos úmidos (alimentos em geral), embalagens, papel, latas, vidros, tecidos, plásticos, entre outros. Devido ao grande volume e a dificuldade no descarte, medidas mitigatórias estão sendo adotadas para melhorar e amenizar os impactos ocasionados pelos resíduos sólidos.

As garrafas de PET pós-consumo foram estudadas e verificou-se que, após o processamento, algumas propriedades são mantidas, favorecendo assim a reutilização desse resíduo como matéria prima (PUROHIT et al., 2012; LÒPEZ et al., 2014). Segundo a Associação Brasileira da Indústria de PET (ABIPET) em 2015 foram reciclados $51 \%$ do total de embalagens em PET produzidas (ABIPET, 2016)

$\mathrm{O}$ material de PET reciclado é destinado às indústrias como matéria prima para desenvolvimento de novos produtos. Atualmente, a indústria têxtil é a detentora de $38 \%$ desse resíduo, $24 \%$ é destinado à indústria de resinas insaturadas e alquídicas, $18 \%$ à indústria de embalagens, $18 \%$ a indústrias de fitas de arquear, laminados e chapas, $2 \%$ à indústria de tubos (Awaja e Pavel, 2005; ABIPET, 2016). Contudo, ainda é limitado o processo de reciclagem do PET devido à escassez de empresas que forneçam material de qualidade.

Geralmente, o PET pós-consumo é comercializado na forma de flakes, os quais apresentam granulometria em torno de 3 a $9 \mathrm{~mm}$, limitando assim sua aplicação em determinados processos (Awaja e Pavel, 2005). Neste sentido, o objetivo desse trabalho é fornecer uma metodologia para moagem do PET com menor granulometria e com maior qualidade.

O PET é considerado um dos mais importantes polímeros de engenharia por apresentar vastas aplicações e excelente resistência à tração e impacto, resistência química e baixa permeabilidade a gases (Awaja e Pavel, 2005).

Dessa forma, o pó de PET de baixa granulometria poderá ser promissor podendo ser agregado a diversos processos produtivos, garantindo assim a sua maior aplicabilidade.

\section{MATERIAL E MÉTODOS}

As garrafas PET pós-consumo utilizadas foram de água mineral com e sem gás. Os rótulos e as tampas foram removidos e descartados. A parte central da garrafa foi lavada com água e sabão, e posta para secagem por 2 horas em temperatura ambiente. Posteriormente, as garrafas foram cortadas em pequenos fragmentos de aproximadamente $10 \times 10 \mathrm{~mm}$.

Cerca de $5 \mathrm{~g}$ dos fragmentos de PET foram colocados em um moinho de panela juntamente com $50 \mathrm{~mL}$ de água aproximadamente. A moagem foi realizada em quatro ciclos de agitação com 1 minuto cada.

Após essa etapa, as amostras foram retiradas da panela do moinho com água e transferidas para um béquer. Com o auxílio de um sistema de filtração a vácuo, a amostra moída foi lavada até apresentar uma coloração clara. A torta de PET gerada foi seca a $60^{\circ} \mathrm{C}$ por 3 horas. A Figura 1 ilustra o processo de fabricação do pó de PET.

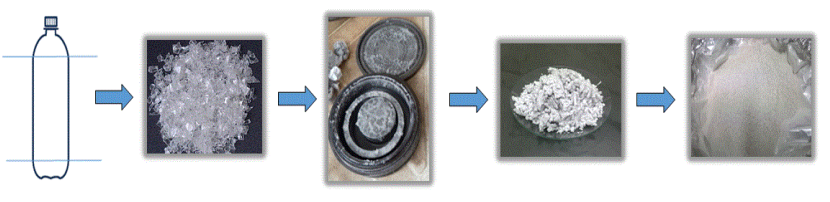

Figura 1 - Processo de fabricação do pó de PET.

O pó de PET produzido foi caracterizado utilizando as técnicas de espectroscopia no infravermelho por transformada de Fourier (FTIR) com a técnica ATR, no aparelho Bruker Alpha, software opus 7.2 em espectrofotômetro Perkin-Elmer. Foi usada a técnica de Calorimetria Diferencial de Varredura (DSC) com calorímetro DSC-60 Shimadzu na faixa de temperatura de 30 a $300{ }^{\circ} \mathrm{C}$ sob fluxo de nitrogênio de 50 $\mathrm{mL} \cdot \mathrm{min}^{-1}$. Termogravimetria (TG) foi realizada usando-se o equipamento DTG60H-SHIMADZU, sendo a massa da amostra de $3,3 \mathrm{mg}( \pm 0,1 \mathrm{mg})$, a razão de aquecimento de $10^{\circ} \mathrm{C} \cdot \mathrm{min}^{-1}$ com intervalo de 20 a $500{ }^{\circ} \mathrm{C}$ e atmosfera dinâmica de $\mathrm{N}_{2}$ com vazão de $10 \mathrm{~mL} \cdot \mathrm{min}^{-1}$. Para o teste granulométrico foram utilizadas as peneiras de 45, 100, 140, 200, 270, 400 Mesh.

\section{RESULTADOS E DISCUSSÃO}

A Figura 2 apresenta o espectro de FTIR da garrafa e pó de PET.

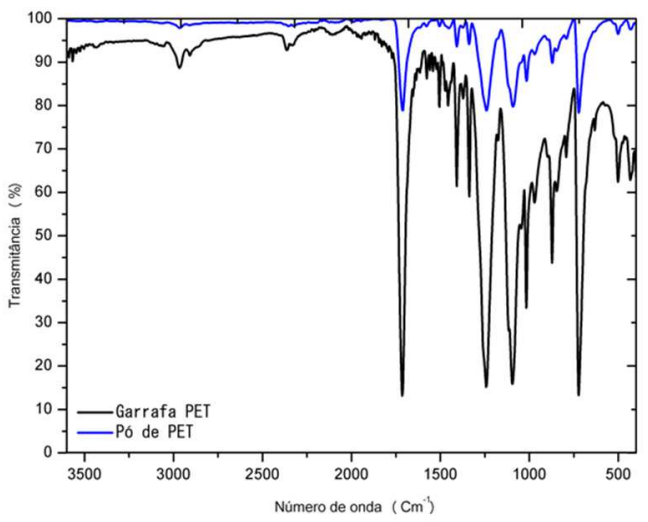

Figura 2 - Espectro de infravermelho da garrafa e pó de PET.

De modo geral, as disposições das bandas estão em conformidade com valores reportados na literatura (CHEN et al., 2012; CHEN et al., 2013; PARVINZADEH et al., 2010). A Tabela 1 apresenta os valores característicos da estrutura química do PET. 
Tabela 1 - Absorção característica de grupos funcionais presentes na garrafa e pó de PET.

\begin{tabular}{|c|c|}
\hline $\begin{array}{c}\text { Número de onda } \\
\left(\mathrm{cm}^{-1}\right)\end{array}$ & Grupo funcional \\
\hline $3200-3600$ & Vibrações de grupos O-H \\
\hline $2850-3000$ & $\mathrm{C}-\mathrm{H}$ alifático \\
\hline $1715-1740$ & $\begin{array}{l}\text { Estiramento de } \mathrm{C}=\mathrm{O} \text { em cetonas não } \\
\text { conjugadas e de grupo éster e/ou de } \\
\text { grupos ácidos carboxílicos }\end{array}$ \\
\hline $1600-1690$ & $\begin{array}{l}\text { Estiramento de } \mathrm{C}=\mathrm{O} \text { de cetonas } \\
\text { aromáticas e aldeídos conjugados }\end{array}$ \\
\hline $1407,1018,1465$ & Deformação assimétrica $\mathrm{CH}_{2-}$ \\
\hline 1242 & Grupo tereftalato $\mathrm{OOC}_{-} \mathrm{C}_{6} \mathrm{H}_{4}-\mathrm{COO}$ \\
\hline $1270-1097$ & Vibração do grupo éster \\
\hline $1043-972$ & Estiramento de grupos éter $\mathrm{C}-\mathrm{O}-\mathrm{C}$ \\
\hline 871,724 & $\begin{array}{l}\text { Vibração =C-H anel benzênico fora } \\
\text { do plano }\end{array}$ \\
\hline
\end{tabular}

A banda de absorção do PET em torno de $1715 \mathrm{~cm}^{-1}$ é atribuída às vibrações do grupo carbonila de ésteres saturados; as bandas em $724 \mathrm{~cm}^{-1}$ e $871 \mathrm{~cm}^{-1}$ são devido à interação de grupos éster polar e anéis de benzeno, vibração $=\mathrm{C}-\mathrm{H}$ fora do plano. A banda de $1097 \mathrm{~cm}^{-1}$ é associada ao modo de vibração de estiramento de ligações $\mathrm{C}-\mathrm{O}$ e a banda em $1242 \mathrm{~cm}^{-1}$ à absorção específica do grupo tereftalato (OOC- $\left.\mathrm{C}_{6} \mathrm{H}_{4}-\mathrm{COO}\right)$.

Os picos referentes à deformação assimétrica em $\mathrm{CH}_{2}$ em torno de $1407 \mathrm{~cm}^{-1}$ e $1018 \mathrm{~cm}^{-1}$ foram identificados. Em $2960 \mathrm{~cm}^{-1}$, observa-se a absorção relativa ao estiramento simétrico da ligação C-H.

De acordo com a literatura, o ponto de fusão $\left(\mathrm{P}_{\mathrm{m}}\right)$ do PET encontra-se em torno de $240-260^{\circ} \mathrm{C}$ (Awaja E Pavel, 2005), conforme apresentado na Figura 3, o valor de $\mathrm{P}_{\mathrm{m}}$ para garrafa e pó foi em torno de $250^{\circ} \mathrm{C}$. A temperatura de transição vítrea $\left(\mathrm{T}_{\mathrm{g}}\right)$ foi de $70^{\circ} \mathrm{C}$ para a garrafa e $72^{\circ} \mathrm{C}$ para o pó (WANG et al., 2000).

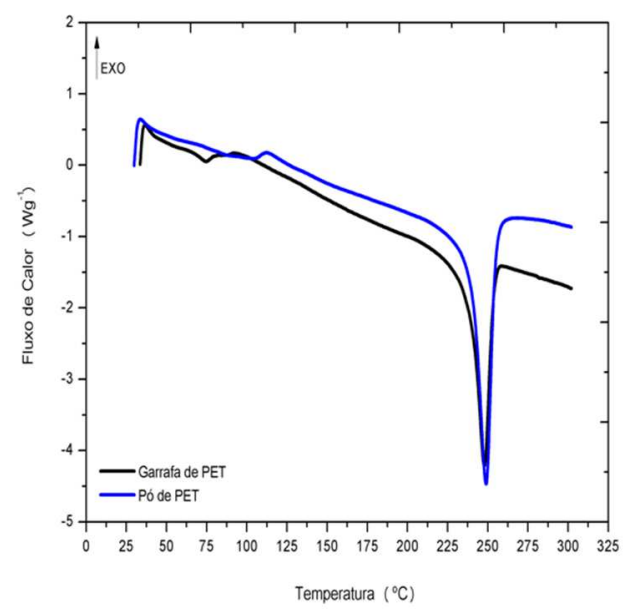

Figura 3 - Curva de DSC da garrafa PET e Pó de PET.

A curva do pó de PET apresentou pico exotérmico acima da $\mathrm{Tg}$, correspondente à temperatura de cristalização $(\mathrm{Tc})$, valor entre 108 e $125^{\circ} \mathrm{C}$.

A temperatura de degradação (Td) foi analisada pelo ensaio de TG. Na Figura 4 (A) e (B) tem-se os valores correspondentes a degradação da garrafa e pó de PET, que foram de $458,9^{\circ} \mathrm{C}$ e $454,7^{\circ} \mathrm{C}$, respectivamente.

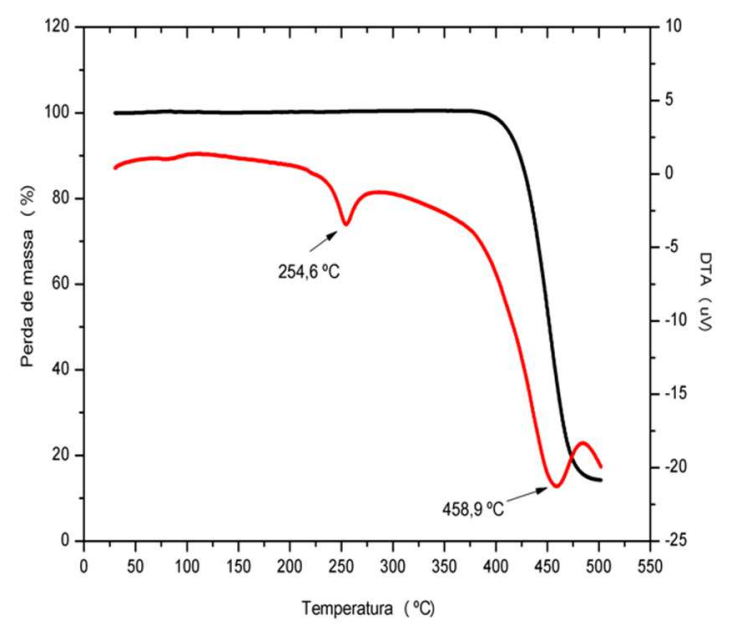

(A)

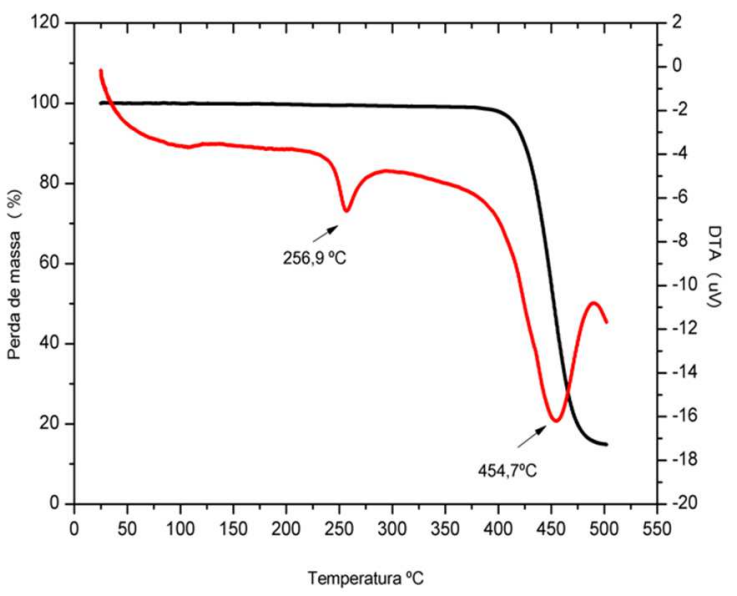

(B) PET (B).

Figura 4 - Análise de TG Garrafa de PET (A) - Pó de

Os tamanhos das partículas do pó de PET foram mensurados pelo teste granulométrico. De acordo com a Tabela 2 , pode-se verificar que a maior quantidade de material ficou retida entre as peneiras de 100 a 200 mesh equivalendo a cerca de $66 \%$.

Tabela 2 - Análise granulométrica do pó de PET.

\begin{tabular}{cccccccc}
\hline $\begin{array}{c}\text { Peneira - } \\
\text { Mesh }\end{array}$ & 45 & 100 & 140 & 200 & 270 & 400 & Fundo \\
\hline $\begin{array}{c}\text { Abertura } \\
(\mathrm{mm})\end{array}$ & 0,354 & 0,149 & 0,105 & 0,074 & 0,053 & 0,037 & - \\
$(\%)$ & 8,0 & 23,4 & 23,0 & 20,4 & 6,8 & 16,4 & 2,0 \\
\hline
\end{tabular}




\section{CONCLUSÕES}

As caracterizações realizadas para a garrafa e pó de PET apresentaram valores similares e em conformidade com os valores da literatura. $\mathrm{O}$ processo de moagem não danificou o polímero. O pó produzido apresentou tamanho entre 0,354 e $0,037 \mathrm{~mm}$, valor bem menor quando comparado com os flakes de PET.

\section{AGRADECIMENTOS}

À CAPES e ao CNPq, pelo auxílio financeiro e ao Sr. Alberto, técnico do laboratório de Minério do Departamento de Engenharia de Minas -UFMG.

\section{R E F E R Ê N C IAS}

ABIPET - Associação Brasileira da Indústria do PET, 2016. Disponível em: < http://www.abipet.org.br/index.html?method=mostrar Downloads\&categoria.id=3>. Acessado em $10 \mathrm{de}$ setembro de 2018.

ABRELPE - Associação Brasileira de Empresas de Limpeza Pública e Resíduos Especiais, 2016. Disponível em: < http://www.abrelpe.org.br/panorama_envio.cfm?ano= 2016>. Acessado em 10 de setembro de 2018.

AWAJA, F.; PAVEL, D. Recycling of PET. European Polymer Journal. v.41, p.1453-1477, 2005.

CHEN, Z., HAY, J.N., JENKINS, M.J. The thermal analysis of poly(ethylene terephthalate) by FTIR spectroscopy. Thermochimica Acta, v.552, p.123-130, 2013.

CHEN, Z.; HAY, J.N.; JENKINS, M.J. FTIR spectroscopic analysis of poly(ethylene terephthalate) on crystallization. European Polymer Journal, v.48, p.1586-1610, 2012.

LÒPEZ, M. D.M.C.; PERNAS, A. I. A.; LÒPEZ, M. J. A.; LATORRE, A. L.; VILARIÑO, J. M. L.; RODRÍGUEZ, M. V. G. Assessing changes on poly(ethylene terephthalate) properties after recycling: Mechanical recycling in laboratory versus postconsumer recycled material. Materials Chemistry and Physics. v.147, p. 884-894, 2014.

PARVINZADEH, M; MORADIAN. S; RASHIDI, A; YAZDANSHENAS, M.E. Surface characterization of polyethylene terephthalate/silica nanocomposites. Applied Surface Science, v.256, p. 2792-2802, 2010.

PUROHIT, J.; CHAWADA, G.; CHOUBISA, B.; PATEL, M.; DHOLAKIYA, B. Polyester Polyol Derived From Waste Poly (Ethylene Terephthalate) for Coating Application on Mild Steel. Chemical Sciences Journal p. 2012, 2012.

WANG, Y.; LU, J.; SHEN, D. Calorimetric and infrared spectroscopic analysis of multiple melting endotherms of poly(ethylene terefhthalate). Polymer Journal, v. 32 , n.7, p. $560-566,2000$. 